\title{
Inverse association of diabetes and dialysis with the severity of femoropopliteal lesions and chronic total occlusion: a cross-sectional study of 2056 cases
}

\author{
Mitsuyoshi Takahara ${ }^{*}$ (D), Yoshimitsu Soga ${ }^{2}$, Masahiko Fujihara ${ }^{3}$, Daizo Kawasaki ${ }^{4}$, Amane Kozuki $^{5}$ \\ and Osamu lida ${ }^{6}$
}

\begin{abstract}
Background: This study aimed to reveal the association of diabetes mellitus and dialysis-dependent renal failure with the lesion severity and chronic total occlusion (CTO) in patients undergoing femoropopliteal endovascular therapy for intermittent claudication.

Methods: This multicenter retrospective study analyzed the data of 2056 consecutive patients with moderate to severe intermittent claudication, who underwent endovascular therapy for de novo lesions in the superficial femoral artery to the proximal popliteal artery between 2010 and 2018 at five cardiovascular centers in Japan. The association of the clinical characteristics with severity of the lesions, as assessed by the Trans-Atlantic Inter-Society Consensus (TASC) II classification, was investigated using the ordinal logistic regression model. Their association with CTO, lesion length, and severity of calcifications was additionally analyzed using the binomial logistic regression model.

Results: The prevalence of diabetes mellitus and dialysis-dependent renal failure was $54.7 \%$ and $21.4 \%$, respectively; $12.5 \%$ of the patients had lesions corresponding to TASC II class D, and $39.3 \%$ of the patients had CTO. Current smoking and severe claudication were associated with more severe lesions assessed according to the TASC II classification; diabetes mellitus and dialysis dependence were inversely associated with disease severity. The adjusted odds ratios of diabetes mellitus and dialysis dependence were 0.82 (95\% confidence interval $0.70-0.97 ; p=0.018$ ) and $0.76(0.62-$ $0.94 ; p=0.009$ ), respectively. Diabetes mellitus and dialysis dependence were also inversely associated with CTO (both $p<0.05)$. Furthermore, diabetes mellitus was inversely associated with a long lesion $(p<0.05)$. Diabetes mellitus and dialysis dependence were positively associated with severe calcification (both $p<0.05$ ).
\end{abstract}

Conclusions: Diabetes mellitus and dialysis-dependent renal failure were inversely associated with the lesion severity, as assessed by the TASC II classification, and CTO in patients undergoing femoropopliteal endovascular therapy for intermittent claudication.

Keywords: Peripheral artery disease, Chronic total occlusion, Diabetes mellitus, Dialysis-dependent renal failure

*Correspondence: takahara@endmet.med.osaka-u.ac.jp

1 Department of Diabetes Care Medicine, Osaka University Graduate

School of Medicine, 2-2 Yamadaoka, Suita City, Osaka 565-0871, Japan

Full list of author information is available at the end of the article

\section{Background}

Patients with diabetes mellitus and renal failure have more severe and complex coronary atherosclerotic disease, including higher rates of chronic total occlusion (CTO), compared to those without these comorbidities [1-4]. The presence of CTO is a strong predictor of poor

(c) The Author(s) 2020. Open Access This article is licensed under a Creative Commons Attribution 4.0 International License, which permits use, sharing, adaptation, distribution and reproduction in any medium or format, as long as you give appropriate credit to the original author(s) and the source, provide a link to the Creative Commons licence, and indicate if changes were made. The images or other third party material in this article are included in the article's Creative Commons licence, unless indicated otherwise in a credit line to the material. If material is not included in the article's Creative Commons licence and your intended use is not permitted by statutory regulation or exceeds the permitted use, you will need to obtain permission directly from the copyright holder. To view a copy of this licence, visit http://creativecommons.org/licenses/by/4.0/. The Creative Commons Public Domain Dedication waiver (http://creativeco mmons.org/publicdomain/zero/1.0/) applies to the data made available in this article, unless otherwise stated in a credit line to the data. 
clinical outcomes among patients undergoing percutaneous coronary intervention $[1,5]$.

The presence of CTO is also a strong predictor of poor clinical outcomes among those undergoing femoropopliteal endovascular therapy for peripheral artery disease (PAD) [6, 7]. However, no clinical studies have examined which comorbidities are associated with complex lesions, especially CTO, in PAD patients. Diabetes mellitus and renal failure are associated with more distally-located (i.e., especially infra-popliteal) arterial disease and more severe calcification [8], but it remains unknown whether the comorbidities are associated with CTO and lesion severity in femoropopliteal segments.

The aim of the current study was to determine the association of diabetes mellitus and dialysis-dependent renal failure with the lesion severity and CTO in patients undergoing femoropopliteal endovascular therapy for intermittent claudication.

\section{Methods}

This multicenter, retrospective study analyzed the data of 2056 consecutive patients between 2010 and 2018 at five cardiovascular centers in Japan, who presented with moderate (Rutherford category 2) to severe (Rutherford category 3) intermittent claudication and underwent endovascular therapy for de novo lesions of the region including the superficial femoral artery to the proximal popliteal artery. The study was conducted in accordance with the Declaration of Helsinki, and was approved by the institutional review boards of the participating institutions. The requirement to obtain any informed consent was waived.

The determination of cardiovascular risk factors was based on the clinical diagnosis according to domestic clinical guidelines. In brief, the presence of hypertension was defined as either (1) having received anti-hypertensive treatment, (2) systolic blood pressure $\geq 140 \mathrm{mmHg}$, or (3) diastolic blood pressure $\geq 90 \mathrm{mmHg}$ [9]. Hyperlipidemia was defined as either (1) having received anti-hyperlipidemic treatment, (2) fasting triglyceride levels $\geq 150 \mathrm{mg} / \mathrm{dl}$, (3) fasting lowdensity lipoprotein cholesterol levels $\geq 140 \mathrm{mg} / \mathrm{dl}$, or (4) non-high-density lipoprotein cholesterol levels $\geq 170 \mathrm{mg} /$ dl [10]. Diabetes mellitus was defined as either (1) having received anti-diabetic treatment, (2) fasting plasma glucose levels $\geq 126 \mathrm{mg} / \mathrm{dl}$, (3) casual plasma glucose levels $\geq 200 \mathrm{mg} / \mathrm{dl}$, or (4) hemoglobin A1c levels $\geq 6.5 \%$ [11]. Dialysis dependence, i.e., end-stage renal disease on dialysis, included both hemodialysis and peritoneal dialysis. Severity of intermittent claudication was classified into moderate (Rutherford category 2) and severe (Rutherford category 3) [12].
The arterial lesions were evaluated based on angiography before endovascular revascularization. Lesion severity was graded according to the Trans-Atlantic Inter-Society Consensus (TASC) II classification [12]. A long lesion was defined as lesion length $\geq 25 \mathrm{~cm}$ [13], and severe calcification was defined as the peripheral arterial calcium scoring system (PACSS) grade 4 [14].

Data are presented as mean \pm standard deviations for continuous variables and as percentages for categorical variables unless otherwise indicated. A two-sided $p$ value $<0.05$ was considered statistically significant. The association of clinical characteristics with the TASC II classification was investigated using the ordinal logistic regression model. We also investigated their association with CTO, long lesions, and severe calcification using the binomial logistic regression model. These associations were presented as odds ratios and $95 \%$ confidence intervals (CIs). All statistical analyses were performed using $\mathrm{R}$ version 3.6.0 ( $\mathrm{R}$ Development Core Team, Vienna, Austria).

\section{Results}

The clinical characteristics of the study population are summarized in Table 1. The prevalence of diabetes mellitus and dialysis-dependent renal failure was $54.7 \%$ and

\section{Table 1 Clinical characteristics of the study population}

\begin{tabular}{ll}
\hline $\boldsymbol{N}$ & $\mathbf{2 0 5 6}$ \\
\hline Male sex & $1490(72.5 \%)$ \\
Age (years) & $73 \pm 9$ \\
Current smoker & $795(38.7 \%)$ \\
Hypertension & $1769(86.0 \%)$ \\
Hyperlipidemia & $1224(59.5 \%)$ \\
Diabetes mellitus & $1125(54.7 \%)$ \\
Dialysis dependence & $441(21.4 \%)$ \\
Diabetes mellitus and dialysis dependence & \\
Diabetes mellitus [-] and dialysis dependence [-] & $764(37.2 \%)$ \\
Diabetes mellitus [-] and dialysis dependence [+] & $167(8.1 \%)$ \\
Diabetes mellitus [+] and dialysis dependence [-] & $851(41.4 \%)$ \\
Diabetes mellitus [+] and dialysis dependence [+] & $274(13.3 \%)$ \\
Severe claudication (Rutherford 3) & $1308(63.6 \%)$ \\
TASC Il classification & \\
Class A & $824(40.1 \%)$ \\
Class B & $371(18.0 \%)$ \\
Class C & $603(29.3 \%)$ \\
Class D & $258(12.5 \%)$ \\
Chronic total occlusion & $807(39.3 \%)$ \\
Lesion length (cm) & $14.3 \pm 9.9$ \\
Lesion length $\geq 25$ cm & $377(18.3 \%)$ \\
Severe calcification & $419(20.4 \%)$ \\
\hline Data are presented as mean & \\
\hline & \\
& \\
\hline
\end{tabular}

Data are presented as mean \pm standard deviation or frequency (percentage) 
$21.4 \%$, respectively; $12.5 \%$ of the patients had TASC II class D lesions, and $39.3 \%$ of the patients had CTO. The current sample size was calculated to be sufficient to detect an adjusted odds ratio of 1.6 (or its reciprocal $1 / 1.6=0.625$ ) between diabetes mellitus or dialysis dependence and respective lesion characteristics, with a statistical power of more than $80 \%$, under an assumption of the observed prevalence and correlation among covariates (Additional file 1: Table S1). As shown in Table 2, current smoking and severity of claudication

Table 2 Association of the clinical characteristics with TASC II classification

\begin{tabular}{lll}
\hline & $\begin{array}{l}\text { Unadjusted odds } \\
\text { ratio }\end{array}$ & Adjusted odds ratio \\
\hline Male sex & $\begin{array}{l}1.04[0.87-1.24] \\
(p=0.68)\end{array}$ & $1.01[0.85-1.21](p=0.89)$ \\
Age (per 10 years) & $\begin{array}{l}0.99[0.90-1.08] \\
(p=0.75)\end{array}$ & $0.96[0.87-1.06](p=0.38)$ \\
Current smoking & $\begin{array}{l}1.25[1.06-1.47] \\
(p=0.007)\end{array}$ & $\begin{array}{l}1.20[1.02-1.42] \\
(p=0.032)\end{array}$ \\
Hypertension & $\begin{array}{l}0.90[0.72-1.13] \\
(p=0.35)\end{array}$ & $0.92[0.73-1.16](p=0.48)$ \\
Hyperlipidemia & $\begin{array}{l}0.95[0.81-1.12] \\
(p=0.53)\end{array}$ & $0.92[0.77-1.08](p=0.31)$ \\
Diabetes mellitus & $\begin{array}{l}0.81[0.70-0.95] \\
(p=0.011)\end{array}$ & $\begin{array}{l}0.82[0.70-0.97] \\
(p=0.018)\end{array}$ \\
Dialysis dependence & $\begin{array}{l}0.78[0.64-0.94] \\
(p=0.010)\end{array}$ & $\begin{array}{l}0.76[0.62-0.94] \\
(p=0.009)\end{array}$ \\
Severe claudication & $\begin{array}{l}1.29[1.10-1.52] \\
(p=0.002)\end{array}$ & $\begin{array}{l}1.31[1.11-1.55] \\
(p=0.001)\end{array}$ \\
\hline
\end{tabular}

Data are presented as odds ratio [95\% confidence interval] ( $p$ value) for TASC II classification. Adjusted odds ratios were derived from the multivariate model in which all the variables listed in the table were entered as the explanatory variables were associated with more severe disease as assessed by TASC II classification, whereas diabetes mellitus and dialysis dependence were inversely associated with disease severity. The adjusted odds ratios of diabetes mellitus and dialysis dependence were 0.82 (95\% CI 0.70-0.97; $p=0.018)$ and 0.76 (95\% CI 0.62-0.94; $p=0.009)$, respectively. No significant interaction effect on the TASC II classification was observed between diabetes mellitus and dialysis dependence $(p=0.98)$. Diabetes mellitus and dialysis dependence were also inversely associated with CTO (Fig. 1a). Furthermore, diabetes mellitus was inversely associated with long lesions (Fig. 1b). By contrast, diabetes mellitus and dialysis dependence were positively associated with severe calcification (Fig. 1c). No significant interaction effect on CTO, long lesions, or severe calcification, was observed between diabetes mellitus and dialysis dependence $(p=0.41,0.33$, and 0.14 , respectively).

\section{Discussion}

Femoropopliteal stenosis or occlusion is the most common lesion associated with intermittent claudication [15]. Currently, endovascular therapy is considered as a first-line revascularization strategy for the lesion $[6,13]$. The current study demonstrated that diabetes mellitus and dialysis-dependent renal failure were inversely associated with lesion severity, as assessed by the TASC II classification, and the presence of CTO in patients undergoing femoropopliteal endovascular therapy for intermittent claudication; meanwhile the two comorbidities were positively associated with calcification. Diabetes mellitus was also inversely associated with long femoropopliteal lesions. No significant interaction effect on lesion characteristics was observed between diabetes mellitus and

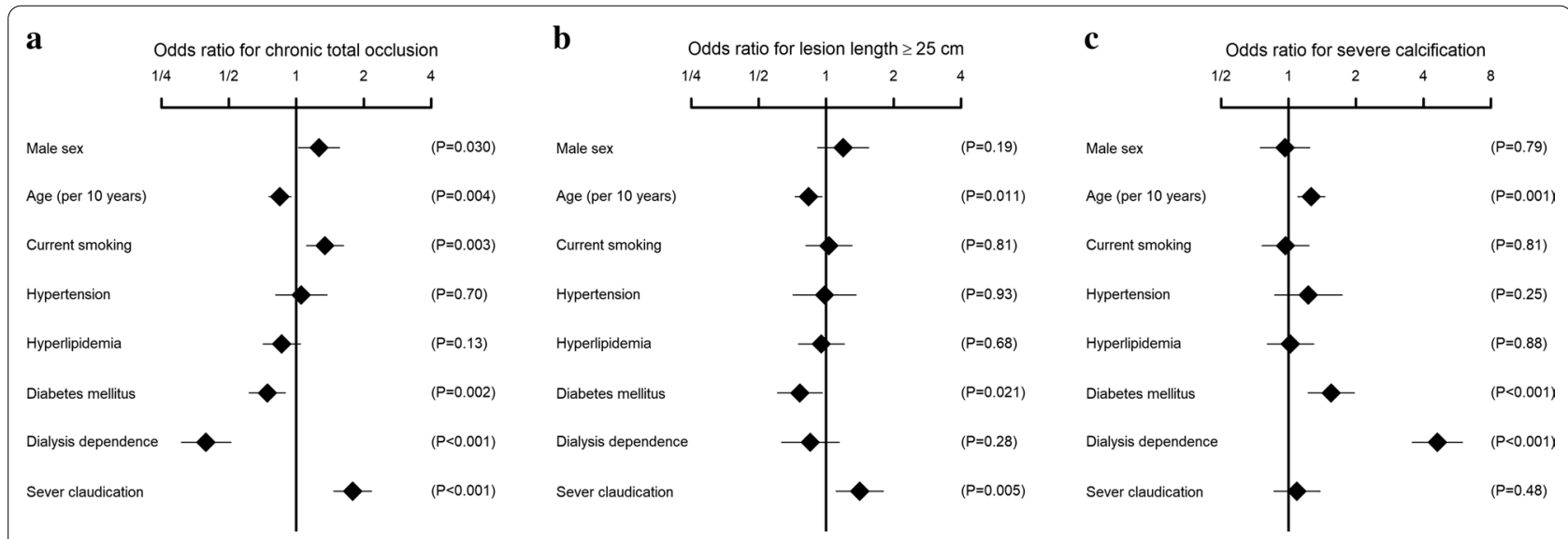

Fig. 1 Association of the clinical characteristics with lesion severity. Data are adjusted odds ratios and 95\% confidence intervals for chronic total occlusion (a), lesion length $\geq 25 \mathrm{~cm}(\mathbf{b})$, and severe calcification (c), derived from the multivariate model in which all the variables listed in the figure were entered as the explanatory variables. Numbers for odds ratios are given in Additional file 1: Table S2 
dialysis dependence, indicating that the impact of diabetes mellitus and dialysis dependence on respective lesion characteristics was additive.

Diabetes mellitus and renal failure are major risk factors for PAD [8, 16, 17]; a high proportion of patients undergoing femoropopliteal endovascular therapy for intermittent claudication have diabetes mellitus and dialysis-dependent renal failure, as seen in clinical practice [18]. Understanding whether patients with these comorbidities have more severe and complex lesions will help interpretating the clinical outcomes of endovascular therapy.

Diabetes mellitus and dialysis-dependent renal failure were found to be positively associated with severe femoropopliteal calcification. Both comorbidities are major accelerators of calcification in coronary and peripheral arteries [12, 19-21]. Our findings regarding femoropopliteal calcification are in line with this data. In contrast, the association of these comorbidities with CTO seems different between coronary and femoropopliteal arteries. In the coronary arteries, diabetes mellitus and renal failure increase the risk of CTO [1-4], whereas our study demonstrated that these comorbidities had an inverse association with femoropopliteal CTO. Furthermore, diabetes mellitus was inversely associated with long femoropopliteal lesions, which is in contrast to the susceptibility of diffuse coronary lesions in patients with diabetes mellitus $[1,2]$. CTO and lesion length are major determinants of lesion severity and complexity. Accordingly, diabetes mellitus and dialysis-dependent renal failure were inversely associated with lesion severity and complexity in femoropopliteal arteries, which was in contrast to the association proved in coronary arteries [1-4].

The pathogenic mechanisms of less severe femoropopliteal lesions in patients with diabetes mellitus and dialysis-dependent renal failure remain unknown. One possible explanation might be the impairment of collateralization. Patients with poor development of collateral vessels might manifest ischemia in the index limb even if occlusive lesions in the main trunk artery are not very severe. Diabetes mellitus is reported to impair the growth of collateral vessels, and various potential mechanisms involving the impairment of arteriogenesis and angiogenesis have been suggested [22]. The contribution of renal failure to impaired collateralization is less clear [23]. Renal failure might have a direct negative effect on collateralization, but also might be a marker of long exposure to uncontrolled diabetes mellitus, since renal failure is a major complication of long-standing diabetes mellitus. Several lines of evidence indicate that, not only impaired vascular flow or perfusion, but also altered skeletal muscle metabolism and inflammatory activation may be responsible for the limb symptoms of PAD [24]. Diabetes mellitus and renal failure might affect these non-vascular mechanistic drivers of claudication [25-28].

Our study had several limitations. First, detailed information about the comorbidities and vessels was limited. No data were available on the etiology of dialysis-dependent renal failure, although we alternatively presented the data on the coexistence of diabetes mellitus and dialysis dependence. Data about the etiology of diabetes mellitus (i.e., type 1 and 2 diabetes mellitus), diabetic neuropathy, and the control of cardiovascular risk factors including diabetes mellitus were also not available. Furthermore, the development of collateral arteries was not assessed since there is no reliable classification system. Second, the current study population was limited to patients with intermittent claudication. It remains unknown whether similar findings were observed in patients with other clinical phenotypes, i.e., asymptomatic patients and those with chronic limb-threatening ischemia. Third, the current study was conducted in Japan. Future studies in other countries are necessary to validate the current findings.

\section{Conclusions}

Diabetes mellitus and dialysis-dependent renal failure were inversely associated with lesion severity, as assessed by the TASC II classification, and CTO in patients undergoing femoropopliteal endovascular therapy for intermittent claudication.

\section{Supplementary information}

The online version contains supplementary material available at https://doi. org/10.1186/s12872-020-01805-6.

Additional file 1: Table S1. Statistical powers. Table S2. Association of the clinical characteristics with lesion severity.

\section{Abbreviations \\ Cl: Confidence interval; CTO: Chronic total occlusions; PACSS: Peripheral arte- rial calcium scoring system; PAD: Peripheral artery disease; TASC: Trans-Atlantic} Inter-Society Consensus.

\section{Acknowledgements}

Not applicable.

\section{Authors' contributions}

MT analyzed data, and MT and OI wrote the manuscript. YS, MF, DK, and AK contributed to the discussion and reviewed/edited the manuscript. All authors read and approved the final manuscript.

\section{Funding}

This study was supported by the Japan Society for the Promotion of Science (JSPS) KAKENHI Grant Number JP18K16199. The funding body played the role in covering the fee for English language editing service and in covering the article processing charge for the journal. The funding entity played no role in the design of the study or in the collection, analysis, or interpretation of data or in writing the manuscript. 


\section{Availability of data and materials}

The data that support the findings of this study are not publicly available due to ethical reasons but are available from the corresponding author upon reasonable request and with permission of the ethics committee of the participating institutions.

\section{Ethics approval and consent to participate}

The study was approved by the Institutional Review Board of Kansai Rosai Hospital (reference number, 16D008g) and the Institutional Review Board of Kokura Memorial Hospital (reference number, 19072452). Because it was a retrospective analysis of existing data, the need for consent was waived by the Institutional Review Board of Kansai Rosai Hospital and the Institutional Review Board of Kokura Memorial Hospital, in accordance with the Ethical Guidelines for Medical and Health Research Involving Human Subjects in Japan.

\section{Consent for publication}

Not applicable.

\section{Competing interests}

The authors declare that they have no competing interests.

\section{Author details}

${ }^{1}$ Department of Diabetes Care Medicine, Osaka University Graduate School of Medicine, 2-2 Yamadaoka, Suita City, Osaka 565-0871, Japan. ${ }^{2}$ Department of Cardiology, Kokura Memorial Hospital, 3-2-1 Asano, Kokurakita-ku, Kitakyushu City 802-0001, Japan. ${ }^{3}$ Department of Cardiology, Kishiwada Tokushukai Hospital, 4-27-1, Kamoricho, Kishiwada City, Osaka 596-8522, Japan. ${ }^{4}$ Cardiovascular Division, Morinomiya Hospital, 2-1-88,Morinomiya, Joto-ku, Osaka City 536-0025, Japan. ${ }^{5}$ Department of Cardiology, Osaka Saiseikai Nakatsu Hospital, 2-10-39, Shibata, Kita-ku, Osaka City 530-0012, Japan. ${ }^{6}$ Cardiovascular Center, Kansai Rosai Hospital, 3-1-69 Inabaso, Amagasaki City, Hyogo 660-8511, Japan.

Received: 27 September 2020 Accepted: 30 November 2020 Published online: 09 December 2020

\section{References}

1. Roffi M, Iglesias JF. CTO PCI in patients with diabetes mellitus: sweet perspectives. JACC Cardiovasc Interv. 2017;10:2182-4.

2. Ledru F, Ducimetiere P, Battaglia S, Courbon D, Beverelli F, Guize L, et al. New diagnostic criteria for diabetes and coronary artery disease: insights from an angiographic study. J Am Coll Cardiol. 2001;37:1543-50.

3. Yan LQ, Guo LJ, Zhang FC, Gao W. The relationship between kidney function and angiographically-derived SYNTAX score. Can J Cardiol. 2011;27:768-72

4. Coskun U, Orta Kilickesmez K, Abaci O, Kocas C, Bostan C, Yildiz A, et al. The relationship between chronic kidney disease and SYNTAX score. Angiology. 2011;62:504-8.

5. Farooq V, Serruys PW, Garcia-Garcia HM, Zhang Y, Bourantas CV, Holmes $D R$, et al. The negative impact of incomplete angiographic revascularization on clinical outcomes and its association with total occlusions: the SYNTAX (Synergy Between Percutaneous Coronary Intervention with Taxus and Cardiac Surgery) trial. J Am Coll Cardiol. 2013;61:282-94.

6. Gerhard-Herman MD, Gornik HL, Barrett C, Barshes NR, Corriere MA, Drachman DE, et al. 2016 AHA/ACC guideline on the management of patients with lower extremity peripheral artery disease: a report of the American College of Cardiology/American Heart Association Task Force on Clinical Practice Guidelines. Circulation. 2017;135:e726-79.

7. Iida O, Takahara M, Soga Y, Fujihara M, Kawasaki D, Hirano K, et al. A novel angiographic risk score for femoropopliteal interventions. J Endovasc Ther. 2020;27:967-73

8. Nativel M, Potier L, Alexandre L, Baillet-Blanco L, Ducasse E, Velho G, et al. Lower extremity arterial disease in patients with diabetes: a contemporary narrative review. Cardiovasc Diabetol. 2018;17:138.

9. Umemura S, Arima H, Arima S, Asayama K, Dohi Y, Hirooka Y, et al. The Japanese society of hypertension guidelines for the management of hypertension (JSH 2019). Hypertens Res. 2019;42:1235-481.
10. Kinoshita M, Yokote $K$, Arai H, lida M, Ishigaki Y, Ishibashi S, et al. Japan atherosclerosis society (JAS) guidelines for prevention of atherosclerotic cardiovascular diseases 2017. J Atheroscler Thromb. 2018:25:846-984.

11. Araki E, Goto A, Kondo T, Noda M, Noto H, Origasa H, et al. Japanese clinical practice guideline for diabetes 2019. Diabetol Int. 2020;11:165-223.

12. Norgren L, Hiatt WR, Dormandy JA, Nehler MR, Harris KA, Fowkes FG, et al. Inter-society consensus for the management of peripheral arterial disease (TASC II). Eur J Vasc Endovasc Surg. 2007;33(Suppl 1):S1-75.

13. Aboyans V, Ricco JB, Bartelink MEL, Bjorck M, Brodmann M, Cohnert T, et al. 2017 ESC Guidelines on the Diagnosis and Treatment of Peripheral Arterial Diseases, in collaboration with the European Society for Vascular Surgery (ESVS): Document covering atherosclerotic disease of extracranial carotid and vertebral, mesenteric, renal, upper and lower extremity arteriesEndorsed by: the European Stroke Organization (ESO)The Task Force for the Diagnosis and Treatment of Peripheral Arterial Diseases of the European Society of Cardiology (ESC) and of the European Society for Vascular Surgery (ESVS). Eur Heart J. 2018;39:763-816.

14. Rocha-Singh KJ, Zeller T, Jaff MR. Peripheral arterial calcification: prevalence, mechanism, detection, and clinical implications. Catheter Cardiovasc Interv. 2014;83:E212-20.

15. White C. Clinical practice. Intermittent claudication. N Engl J Med. 2007:356:1241-50.

16. Murabito JM, D'Agostino RB, Silbershatz H, Wilson WF. Intermittent claudication. A risk profile from The Framingham Heart Study. Circulation. 1997;96:44-9.

17. Wattanakit K, Folsom AR, Selvin E, Coresh J, Hirsch AT, Weatherley BD. Kidney function and risk of peripheral arterial disease: results from the Atherosclerosis Risk in Communities (ARIC) Study. J Am Soc Nephrol. 2007;18:629-36.

18. Takahara M, lida O, Kohsaka S, Soga Y, Fujihara M, Shinke T, et al. Diabetes mellitus and other cardiovascular risk factors in lower-extremity peripheral artery disease versus coronary artery disease: an analysis of 1,121,359 cases from the nationwide databases. Cardiovasc Diabetol. 2019:18:155.

19. Giachelli CM. Vascular calcification mechanisms. J Am Soc Nephrol. 2004;15:2959-64

20. Schurgin S, Rich S, Mazzone T. Increased prevalence of significant coronary artery calcification in patients with diabetes. Diabetes Care. 2001;24:335-8.

21. Nakamura S, Ishibashi-Ueda H, Niizuma S, Yoshihara F, Horio T, Kawano Y. Coronary calcification in patients with chronic kidney disease and coronary artery disease. Clin J Am Soc Nephrol. 2009;4:1892-900.

22. Shen $Y$, Ding FH, Dai Y, Wang XQ, Zhang RY, Lu L, et al. Reduced coronary collateralization in type 2 diabetic patients with chronic total occlusion. Cardiovasc Diabetol. 2018;17:26

23. Xie SL, Li HY, Deng BQ, Luo NS, Geng DF, Wang JF, et al. Poor coronary collateral vessel development in patients with mild to moderate rena insufficiency. Clin Res Cardiol. 2011;100:227-33.

24. Hamburg NM, Creager MA. Pathophysiology of intermittent claudication in peripheral artery disease. Circ J. 2017:81:281-9.

25. Zabielski P, Lanza IR, Gopala S, Heppelmann CJ, Bergen HR 3rd, Dasari $\mathrm{S}$, et al. Altered skeletal muscle mitochondrial proteome as the basis of disruption of mitochondrial function in diabetic mice. Diabetes. 2016:65:561-73.

26. Workeneh BT, Mitch WE. Review of muscle wasting associated with chronic kidney disease. Am J Clin Nutr. 2010;91:1128S-S1132.

27. Donath MY, Shoelson SE. Type 2 diabetes as an inflammatory disease. Nat Rev Immunol. 2011;11:98-107.

28. Pecoits-Filho R, Heimburger O, Barany P, Suliman M, Fehrman-Ekholm I, Lindholm B, et al. Associations between circulating inflammatory markers and residual renal function in CRF patients. Am J Kidney Dis. 2003;41:1212-8.

\section{Publisher's Note}

Springer Nature remains neutral with regard to jurisdictional claims in published maps and institutional affiliations. 